\title{
A Modified Enhanced Recovery Pathway after Pancreaticoduodenectomy: Full Liquids on Discharge
}

\author{
George Younan ${ }^{1,2}$, Archana Jindal ${ }^{3}$, Maged Andrews ${ }^{3}$, Danielle Eganhouse ${ }^{2}$ and Timothy R. Shaver ${ }^{1,2}$ \\ ${ }^{1}$ Department of Surgery, Inova Fair Oaks Hospital, Fairfax, VA. \\ ${ }^{2}$ Division of Hepato-Pancreato-Biliary Surgery, Virginia Surgery Associates, Fairfax, VA. \\ ${ }^{3}$ Department of Anesthesiology, North American Partners in Anesthesia, Inova Fair Oaks Hospital, Fairfax, VA.
}

\begin{abstract}
Background: After success in many surgical disciplines, enhanced recovery after surgery pathways have been recently introduced to the care of patients after pancreatic surgery. The aim of this study is to share our improved outcomes with a modified pathway that allows patients to be discharged home on a full liquid diet.

Methods: A modified enhanced recovery pathway was based on published guidelines but included new changes aiming to improve outcomes after pancreaticoduodenectomy in a total of 60 consecutive patients.

Results: Patients included in this study had no significant differences in demographics and preoperative characteristics. By implementing a modified enhanced recovery protocol, we were able to improve post-operative parameters, including a decrease in the length of stay to a median of 5 days. Compliance rate with the program reached $88 \%$ and patients were able to maintain a similar weight compared to the regular pathway at 30 days. Time to adjuvant therapy was reduced without significantly increasing overall complications or readmission rates.
\end{abstract}

Conclusion: A modified enhanced recovery pathway after pancreaticoduodenectomy successfully improved short-term outcomes and reduced hospital stay.

\section{KEYWORDS}

Surgery, Pancreaticoduodenectomy, Pancreatic fistula.

Corresponding Author Information

George Younan, MD,

Virginia Surgery Associates, 3620 Joseph Siewick Drive \#406, Fairfax, VA 22033, Tel: 703-359-8640; Fax: 703-591-6105.

Received: August 29, 2020; Accepted: September 14, 2020; Published: September 19, 2020

Copyright: (C) 2020 ASRJS. This is an openaccess article distributed under the terms of the Creative Commons Attribution 4.0 International license.

Citation: George Younan, Maged Andrews, Umadevi Rangarajan, Danielle Eganhouse, Robert F. Ahmed, et al. Improved Pancreaticoduodenectomy Outcomes after Starting a Specialized Community Hospital Pancreatic Surgery Program. Med Clin Res Open Access. 2020;1(1):1-7. 


\section{Introduction}

The Whipple procedure, or pancreaticoduodenectomy (PD) remains one of the most complex abdominal surgical procedures, requiring superior surgical skills and resulting in high morbidity rates [1]. Even in high volume centers and in the hands of high volume surgeons, recovery from this procedure is still a lengthy process [2]. While 30-day mortality rates have reached a low of $1-2 \%$ in specialized centers, morbidity rates remain in the range of $40-60 \%[3,4]$. Hospital length of stay (LOS) after PD, in the absence of complications, remains in the range of seven to eleven days in the United States and much higher internationally [5]. Postoperative pancreatic fistula (POPF), delayed gastric emptying (DGE) and other complications add additional delay to recovery $[3,6]$.

In the face of increased health care costs, there has been a national push towards centralization of care in order to decrease mortality and morbidity, hence reducing health care expenditure on pancreatic resections [7-10]. Extended indications for pancreatic resections are being utilized now, increasing the number of PD performed annually for benign and malignant diagnoses and locally advanced tumors that did not get surgically treated previously $[11,12]$. Thus, new perioperative protocols were needed to meet all these requirements.

Enhanced recovery after surgery (ERAS) pathways are processes aimed to improve perioperative care and outcomes after surgical procedures [13]. Initially introduced by Kehlet et. al in 1997, these pathways, that started mainly in colorectal surgery, are now being used among many surgical disciplines [14]. ERAS in PD was slow to be adopted due to the complexity of its perioperative care and the high likelihood of morbidity and events that might skew any standardized pathway [15]. Guidelines were published by the ERAS society in 2012 to serve as roadmap for pancreatic surgeons to use [13]. In recent years, many variations of "fast track" recovery after PD have been published [5,16-20]. A main target in the majority of these pathways is reduction of hospital LOS after a PD without increasing 30-day readmissions and complications [5].

We have developed a modified ERAS protocol for our PD cases and were able to decrease the LOS to a median of five days. We believe that in addition to excellent surgical technique as a predictor for good outcomes, a significant reduction of LOS can be obtained by discharging patients home once they tolerate a full liquid diet. Our decision to use full liquids on discharge was extrapolated from bariatric and foregut surgery data where this diet is used. We describe herein our modified protocol and our outcomes before and after its implementation.

\section{Methods}

A prospectively-maintained database was used to analyze data in a retrospective fashion.

The database was saved at the division of hepatopancreatobiliary surgery at Virginia Surgery Associates (Fairfax, VA). Patients who underwent PD surgery with the two pancreatic surgeons of the group (GY and TRS), were identified and their charts reviewed. Patients demographics, preoperative, intraoperative and postoperative parameters were recorded. Data from August 2016, when a fellowship-trained pancreatic surgeon (GY), joined the group and a pancreatic surgery program was collected. Sixty consecutive PD were performed in a period of twenty-eight months, until December of 2018. An ERAS protocol was introduced in October of 2017 and this was exclusively used for the rest of the study period, based on favorable outcomes. A total of 27 patients underwent a PD before ERAS and 33 patients after it, for a total of 60 patients in this study.

The surgical technique was consistent in all cases. Both surgeons were present in the majority of cases and the reconstruction was exclusively done by one surgeon (GY) based on published steps [21]. We performed the standard PD procedure without preserving the pylorus. A duct-to-mucosa retrocolic end-to-side pancreaticojejunostomy was exclusively performed in all cases. One 19 French blake drain was placed in the majority of cases in the right paracolic gutter posterior and lateral to both biliary and pancreatic anastomoses, another anterior drain was used only twice in the whole cohort based on surgeon preference. The drain amylase was not measured in any case and drain removal was standardized on patients first post-operative visit to the hepatobiliary clinic in the majority of cases. Post-operative care was standardized for all cases and an ERAS pathway was used after October of 2017.

Antibiotics prophylaxis was used in all cases and kept for three to five consecutive days depending on post-operative white blood cell counts and bile culture results. Deep vein thrombosis prophylaxis was performed with compression devices and chemical prophylaxis was not used until post-operative day number one. Octreotide subcutaneous injections were prophylactically used in all cases before the ERAS protocol was implemented and never used after that. A multimodality pain pathway protocol was developed in conjunction with the anesthesia department. Nerve blocks were used in a minority of cases initially and the drug of choice was nonliposomal Bupivacaine, however after ERAS, all patients received nerve blocks with liposomal Bupivacaine.

All patients were followed by an acute pain service postoperatively as they all received intravenous narcotics through a patient-controlled analgesia pump until they were switched to oral narcotics once started on a liquid diet. All patients were given scheduled acetaminophen and gabapentin around the clock postoperatively. Patients were admitted to monitored hepatobiliary unit beds after their procedures and did not need intensive care unit stay unless indicated (Table 1). Shows the comparison of the pre and post-ERAS pathway protocols. Patients nasogastric tubes were left until output was less than $500 \mathrm{ml} / 24 \mathrm{hrs}$ initially in the regular pathway group, diets were advanced thereafter on a daily basis up to a low residue diet.

After ERAS, all nasogastric tubes were left in for a standard of three days, all patients received a clear liquid diet on POD\#3 and advanced to a full liquid diet on POD\#4 and stayed on that diet 
when discharged home. All patients received additional highcalorie, high-protein liquid supplements on a daily basis. Patients diets were advanced to low residue diets when they were seen in the office for their weekly, and sometimes biweekly post-operative visit, once ready for that. We allowed patients to participate in that decision during regular phone call checkups after discharge. Patients were kept on oral promotility agents after discharge.

\begin{tabular}{|c|c|c|}
\hline & Regular Pathway & ERAS Pathway \\
\hline \multicolumn{3}{|l|}{ Pre-operative } \\
\hline Tumor board & None & $\begin{array}{l}\text { Multidisciplinary } \\
\text { biweekly meeting }\end{array}$ \\
\hline Bowel preparation & None & $\begin{array}{c}\text { Miralax bowel } \\
\text { preparation }\end{array}$ \\
\hline $\begin{array}{l}\text { Preoperative } \\
\text { medications }\end{array}$ & None & $\begin{array}{c}\text { Acetaminophen and } \\
\text { Gabapentin }\end{array}$ \\
\hline $\begin{array}{l}\text { Nerve block pre- } \\
\text { incision }\end{array}$ & Rarely used & $\begin{array}{l}\text { Always with } \\
\text { Liposomal } \\
\text { Bupivacaine }\end{array}$ \\
\hline \multicolumn{3}{|l|}{ Intra-operative } \\
\hline Intraoperative fluids & As needed policy & Restrictive policy \\
\hline $\begin{array}{c}\text { Intraoperative } \\
\text { narcotics }\end{array}$ & As needed policy & Restrictive policy \\
\hline \multicolumn{3}{|l|}{ Post-operative } \\
\hline $\begin{array}{l}\text { Nasogastric tube } \\
\text { (NGT) }\end{array}$ & $\begin{array}{c}\text { Depending on } \\
\text { output }\end{array}$ & $\begin{array}{l}\text { Removal POD\#3 if } \\
\text { drainage }<500 \mathrm{cc}\end{array}$ \\
\hline Promotility agents & As needed & $\begin{array}{l}\text { Scheduled Reglan } \\
\text { starting POD\#2 }\end{array}$ \\
\hline Oral clear liquids & $\begin{array}{c}\text { Once NGT } \\
\text { removed }\end{array}$ & POD\#3 \\
\hline Oral low residue food & $\begin{array}{c}\text { Once clears are } \\
\text { tolerated }\end{array}$ & After discharge \\
\hline Pain pathway & As needed & Always \\
\hline Octreotide & Always & None \\
\hline $\begin{array}{l}\text { Supplemental } \\
\text { nutrition }\end{array}$ & Most cases & None \\
\hline Mobilization & Ad lib & Walking program \\
\hline
\end{tabular}

Table 1: Comparison of regular and ERAS pathways.

The steps of the modified ERAS protocol were printed on large boards and placed in patient rooms, as to set expectations for patients, their families and nursing staff. Patients were discharged home after meeting several criteria including: the absence of complications, comfort of patients of being discharged home, tolerance of a full liquid diet and absence of the need of intravenous medications. Passage of bowel movements was not a criterion for discharge in the absence of obvious signs of post-operative ileus.

Complications were measured using published guidelines from the International Study Group on Pancreatic Surgery and the Pancreas Club calculators [22-24]. The international definition of DGE was partially applied as our patients were not advanced to a regular diet before discharge. We relied only on NGT days or reinsertion after surgery. Patients who had their tubes for 4-7 days were grade A and 8-14 days were grade B. We did not have grade C DGE in our cohort. Patient outcomes were compared using standard statistical methods. We compared outcomes of surgeries before and after ERAS was implemented, upon approval from the institutional review board as an outcomes study. Statistical analysis was done using GraphPad Prism (Lajolla, CA) and p-value $<0.05$ was considered significant.

\section{Results}

Sixty consecutive PD were done by our new pancreatic surgery program from August 2016 to December 2018. The ERAS pathway was introduced in October of 2017 and was exclusively used thereafter. Twenty-seven PD were done before and thirty-three after the implementation of the program.

There was no difference in patient demographics before and after ERAS was implemented and this is shown in Table 2. Patients were of similar age (64 vs, 63 years), gender ( 66 vs. $60 \%$ males), body mass index ( 26.1 vs. $26.3 \mathrm{~kg} / \mathrm{m} 2)$ and American Society of Anesthesiologists (ASA) class. Additionally, indications for surgery were not significantly different, $74 \%$ were done for a cancer diagnosis, $13 \%$ for a pre-malignant lesion and $13 \%$ for chronic pancreatitis.

Intraoperative results and post-operative outcomes are shown in Table 2. After implementation of the ERAS program, there was a significant decrease in surgical operative time (344 and 273 minutes, $\mathrm{p}<0.01)$ and estimated blood loss of the procedure $(312$ and $146 \mathrm{ml}, \mathrm{p}<0.01$ ). There a significant difference in the use of liposomal Bupivacaine nerve blocks after ERAS and all patients received that before surgical incision, whereas only $30 \%$ of patients received a nerve block before ERAS and these received the regular non-liposomal Bupivacaine.

Post-operative blood transfusions were reduced from $7.4 \%$ to $3 \%$ however these results did not reach statistical significance. There was no difference in pancreatic gland texture or pancreatic duct diameter between patients. These features increase the complexity of reconstruction after PD and are known risk factors for postoperative pancreatic fistulae [25].

Overall delayed gastric emptying was reduced from $14.8 \%$ to $6 \%$, equally divided between grades A and B. There was no Grade C delayed gastric emptying in this study. Pancreatic leak rates were similar between the two hospitals; there was one Grade B leak and one Grade C leak before ERAS, versus one grade B and no Grade $\mathrm{C}$ leaks after ERAS. There was no difference in intra-abdominal infections, which included any abscesses or fluid collections that needed to be treated with percutaneous drains or antibiotics and these included all pancreatic fistulae, ( $11 \%$ and $6 \%, \mathrm{p}=0.65)$.

We had no deep vein thrombosis or pulmonary emboli, all patients received mechanical prophylaxis during and after surgery and chemical prophylaxis on post-operative day one. Only one patient 
after ERAS had a urinary tract infection requiring treatment with antibiotics. R0 resection rates were excellent and similar between the two groups (92\% and 97\%).

Length of hospital stay was significantly reduced after ERAS from 7.1 to 5.2 days $(\mathrm{p}<0.01$ ). This resulted in an increase in 30 -day readmission rates from $7.4 \%$ to $15 \%$. Patients were readmitted different reasons, three of them for social reasons and having no support at home. We had one reoperation for sepsis and multiorgan failure and that one case was also the only 30 -day mortality.

When we followed patients for 30 days, we measured their percent body weight change, all patient weights were lower than their presurgery weights, however there was no difference between the two groups. Patients who were sent home on a full liquid diet did not lose more weight compared to patients who were advanced to a regular diet before discharge $(.6 .6 \%$ vs. $-6.8 \%, \mathrm{p}=0.84)$. Patients on the ERAS pathway were able to start adjuvant therapy when indicated significantly earlier than those before the program was implemented, (62 vs. 44 days, $\mathrm{p}<0.01$ ).

\begin{tabular}{c|c|c|c}
\hline & Pre-ERAS & Post-ERAS & p value \\
\hline Number of Procedures & 27 & 33 & \\
\hline Age (Median, Years) & 64 & 63 & 0.78 \\
\hline Male Gender (\%) & $18(66 \%)$ & $20(60 \%)$ & 0.83 \\
\hline BMI (kg/m $)^{2}$ & 26.1 & 26.3 & 0.9 \\
\hline ASA Class & & & 0.36 \\
\hline 2 & 4 & 6 & \\
\hline 3 & 13 & 19 & \\
\hline 4 & 10 & 8 & \\
\hline Preop Total Bilirubin & 3.1 & 4.9 & 0.2 \\
\hline Preop Albumin & 3.1 & 3.2 & 0.52 \\
\hline Preop Biliary Stent & $13(48 \%)$ & $17(51 \%)$ & 1 \\
\hline Indication of Procedure & & & 0.76 \\
\hline Malignancy & 21 & 23 & \\
\hline Pre-malignancy & 2 & 6 & \\
\hline Pancreatitis & 4 & 4 & \\
\hline
\end{tabular}

Table 2: Patient demographics and preoperative variables.

\begin{tabular}{|c|c|c|c|c|}
\hline & & Pre-ERAS & Post-ERAS & p value \\
\hline \multicolumn{2}{|c|}{ Operative Time (Minutes) } & 344 & 273 & 0.0001 \\
\hline \multicolumn{2}{|c|}{ Estimated Blood Loss (ml) } & 312 & 146 & 0.0002 \\
\hline \multicolumn{2}{|c|}{ Liposomal Anesthetic Nerve Block } & $8(29.6 \%)$ & $33(100 \%)$ & 0.01 \\
\hline \multicolumn{2}{|c|}{ PD Diameter (Median, mm) } & 3 & 4 & 0.54 \\
\hline \multicolumn{2}{|c|}{ Gland Texture (Soft \%) } & $17(63 \%)$ & $16(49 \%)$ & 0.66 \\
\hline \multicolumn{2}{|c|}{ Tube Feeds or TPN } & $16(59 \%)$ & $0(0 \%)$ & 0.0001 \\
\hline \multirow{3}{*}{ Delayed Gastric Emptying } & Grade A & $2(7.4 \%)$ & $1(3.0 \%)$ & \multirow{3}{*}{0.4} \\
\hline & Grade B & $2(7.4 \%)$ & $1(3.0 \%)$ & \\
\hline & Grade C & 0 & 0 & \\
\hline \multicolumn{2}{|c|}{ Post-operative Blood Transfusion } & $2(7.4 \%)$ & $1(3.0 \%)$ & 0.59 \\
\hline \multirow{2}{*}{ Pancreatic Leak } & Garde B & $1(3.7 \%)$ & $1(3.0 \%)$ & \multirow{2}{*}{0.59} \\
\hline & Grade C & $1(3.7 \%)$ & $0(0 \%)$ & \\
\hline \multicolumn{2}{|c|}{ Intra-abdominal Infection } & $3(11.1 \%)$ & $2(6 \%)$ & 0.65 \\
\hline \multicolumn{2}{|c|}{ ICU Stay Requirement } & $3(11.1 \%)$ & $3(9 \%)$ & 1 \\
\hline \multicolumn{2}{|c|}{ R0 Resection } & $25(92 \%)$ & $32(97 \%)$ & 1 \\
\hline \multicolumn{2}{|c|}{ DVT/PE/UTI } & $1(3.7 \%)$ & $1(3.0 \%)$ & 1 \\
\hline \multicolumn{2}{|c|}{ Length of Hospital Stay (Days) } & 7.1 & 5.2 & 0.0002 \\
\hline \multicolumn{2}{|c|}{ 30-day Readmission } & $2(7.4 \%)$ & $5(15 \%)$ & 0.69 \\
\hline \multicolumn{2}{|c|}{ Reoperation } & $1(3.7 \%)$ & $0(0 \%)$ & 0.45 \\
\hline \multicolumn{2}{|c|}{ 30-day Mortality } & $1(3.7 \%)$ & $0(0 \%)$ & 0.45 \\
\hline \multicolumn{2}{|c|}{ Percent Change in Body Weight at 30 Days } & $-6.60 \%$ & $-6.80 \%$ & 0.84 \\
\hline \multicolumn{2}{|c|}{ Time to Adjuvant Therapy (Days) } & 62 & 44 & 0.0026 \\
\hline
\end{tabular}

Table 3: Postoperative outcomes. 


\section{Discussion}

The pancreatic surgery program was initiated after a fellowshiptrained pancreatic surgeon (GY), joined an already established hepatobiliary surgeon (TRS) in Northern Virginia. In October of 2017, both surgeons implemented a new modified ERAS protocol for PD based on both of their training preferences. This study compares procedures done since the start of the program and until ERAS was introduced, to the procedures done after that. Outcomes of the program improved with time, due to many factors, one of them is the learning curve of the newly trained surgeon (GY), however we believe that the ERAS pathway had a major contribution to many of our short-term post-operative outcomes. The components of the pathway were a combination of published literature, in addition to our surgeon preference that reflects their respective training $[13,17,26]$. The majority of pancreatic surgeries in this program were done in a specialized community center that is a center of excellence in bariatric surgery. Most bariatric surgery patients were sent home in 24-48 hours after their procedures on a liquid diet.

The pancreatic surgery program patients shared the same surgical ward with those patients and nurses were very comfortable with an already established pathway. We decided to use that same pathway for our PD patients, knowing that a liquid diet offers more calories per volume compared to a solid diet. To our surprise, the rates of nausea after surgery dropped significantly and patients were very comfortable going home on that type of diet. In preparation for discharge, patients and their families were educated on the types of liquids to prepare and stock at home, in addition to high-calorie, high-protein drinks and electrolyte-rich juices at home. All patients were given an oral form of a promotility agent to take and this was reassessed on their first clinic visit after discharge. With our protocol we were able to eliminate the use of enteral or parenteral nutrition at discharge. Delayed gastric emptying was reduced from $15 \%$ to $6 \%$ using this program, these results were not statistically significant. However, clinically speaking, our protocol was very successful in reducing nausea symptoms and allowing patients to be discharged earlier as long as their full liquid diet is tolerated.

A key factor to these results is the combination of full liquids and a promotility agent. The two patients who had vomiting after ERAS were the ones who failed to take promotility agents at home. Of note, once they restarted that, all symptoms resolved and they resumed their diets. We were able to treat one of them while at home and the other patient had to be readmitted for hydration for less than one day. When we followed our patients for 30 days, we were able to prove the safety of full liquids on discharge. Percentage body weight change at 30 days was similar between the two groups, (-6.6\% and-6.8\%), knowing that $60 \%$ of patients from the conventional pathway additionally received either enteral or parenteral nutrition after discharge to home.

There is one main study in the literature where investigators used full liquids on discharge, this was published by Lavu et. al in 2018, and in their study, which was happening around the same time of our study, patients were also advanced to a regular diet while at home through a telehealth follow-up program. They were able to send $75 \%$ of their patients home on POD\#5 while using this pathway [5].

Our pathway led to decrease in the LOS after PD from 7.1 to 5.2 days, and this is one of the lowest averages in hospital stay that we could find in the literature. All major enhanced pathways after a PD aimed to discharge patients in 5 days $[5,16]$. The percentages of patients who were discharged home on POD $\# 5$ in the literature ranges from 60 to $80 \%$ in general $[5,16]$. Patients who were able to be discharged home on or before POD\#5 were deemed "highperformers" in a study by Lee et. al. [3] We were able to discharge $88 \%$ of our patients' home on POD\#5 after PD.

When studying the factors leading to the success of the ERAS pathway, multiple interventions were used in combination allowing improved outcomes. First is in the preoperative stage, where a multidisciplinary team is involved in patient selection and all decisions and expectations are shared with patients during the planning part of the treatment. Patients are involved in planning their early discharge, and dates of surgery and dates of discharge home are set and known. Food and drink supplies are made ready at home, days before discharge home [13].

Second is an efficient and robust perioperative stage, starting the morning of surgery, going through goal-directed intraoperative fluid and narcotic management by an experienced anesthesia team and ending with a good surgical technique, minimizing procedurerelated complications like intraoperative blood loss requiring blood transfusions, pancreatic anastomotic leaks and length of the surgical procedures $[20,27]$. These intraoperative variables directly influence short-term outcomes like DGE and LOS after pancreatic surgery $[17,28-30]$.

Third is a well-planned recovery pathway after surgery that will help drive and improve outcomes once other technique-related morbidities are prevented [19]. Delaying oral feeding to POD\#3 in our model was used for a variety of reasons. Debate regarding the best nasogastric decompression strategy continues and different institutions use different strategies [31,32]. We believe that after a Billroth-II type reconstruction of the gastrojejunostomy anastomosis after $\mathrm{PD}$, decompression helps decreasing the intraluminal pressure in the afferent jejunal limb draining both pancreatic and biliary anastomoses and thus we needed to safely maximize decompression for at least three days. It is also believed that delaying oral feeding reduces the rate of postoperative ileus and allows the freshly-constructed gastrojejunostomy to have less swelling and inflammation allowing improved liquid transit [5].

A very important aspect of the pathway is that active care by our team continued after discharge with a very close phone-based follow up. Active management of patients does not end when they are discharged home, and in fact, the major part of functional and physical recovery happens at home in their baseline environment. Many variables play into the decision of starting adjuvant chemotherapy after PD for cancer patients. Recovery from PD 
is a lengthy process and the majority of patients need weeks to months before reaching an acceptable performance status before embarking on another debilitating type of systemic treatment. Enhanced recovery plays a crucial role in improving overall oncological outcomes once systemic therapy can be started earlier. We were able to reduce time to adjuvant therapy from 62 to 44 days after $\mathrm{PD}$, and this is ab acceptable duration when compared to national benchmarks. 5 We believe that a shorter wait can be achieved through multidisciplinary teams that involve medical oncologists early on during the overall treatment process.

This study has several limitations, including the low number of patients in each group, in addition to its retrospective nature. After ERAS was implemented, and after the team solidified its presence and leadership in the community program, all variables were strictly regulated. This was not the case before ERAS and there was room for variations, per example, the decision to insert a jejunostomy tube or to send patients home on total parenteral nutrition. An additional limitation is the switch from an academic program where residents were involved in the care of these patients early in the study, to a community program that did not involve residents. Using liposomal Bupivacaine was also one element that changed throughout the implementation of the pathway, whether or not it had an effect in decreasing narcotics use, DGE and LOS is still to be determined in a future study. A relative limitation of the study is the low numbers of complex PD that required vascular resections and the low percentage of patients who were given neoadjuvant therapy and that was less than ten percent of the cases.

This study adds to the growing body of evidence showing that care of post-PD patients can be safely done at home after recovering for 5 days at the hospital $[5,33]$. A full liquid diet on discharge is safe and confers adequate nutritional value [34]. The safety of this diet on discharge should act as an incentive for larger institutions to adopt this pathway and advocate for a modification in the international guidelines definition of delayed gastric emptying as not to include tolerance of solid food as a requirement.

\section{Conclusion}

A modified ERAS pathway after PD successfully reduced hospital stay and improved short-term recovery. Patients had reduced rates of DGE symptoms and were able to recover faster and start adjuvant therapy earlier.

\section{References}

1. Cameron JL, He J. Two thousand consecutive pancreaticoduodenectomies. J Am Coll Surg. 2015;220(4):530536.

2. Enomoto LM, Gusani NJ, Dillon PW, Hollenbeak CS. Impact of surgeon and hospital volume on mortality, length of stay, and cost of pancreaticoduodenectomy. J Gastrointest Surg. 2014;18(4):690-700.

3. Lee GC, Fong ZV, Ferrone CR, et al. High performing whipple patients: factors associated with short length of stay after open pancreaticoduodenectomy. J Gastrointest Surg.
2014;18(10):1760-1769.

4. DeOliveira ML, Winter JM, Schafer M, et al. Assessment of complications after pancreatic surgery: A novel grading system applied to 633 patients undergoing pancreaticoduodenectomy. Ann Surg. 2006;244(6):931-937; discussion 937-939.

5. Lavu H, McCall NS, Winter JM, et al. Enhancing Patient Outcomes while Containing Costs after Complex Abdominal Operation: A Randomized Controlled Trial of the Whipple Accelerated Recovery Pathway. J Am Coll Surg. 2019.

6. Eisenberg JD, Rosato EL, Lavu H, Yeo CJ, Winter JM. Delayed Gastric Emptying After Pancreaticoduodenectomy: an Analysis of Risk Factors and Cost. J Gastrointest Surg. 2015;19(9):1572-1580.

7. Stitzenberg KB, Sigurdson ER, Egleston BL, Starkey RB, Meropol NJ. Centralization of cancer surgery: implications for patient access to optimal care. J Clin Oncol. 2009;27(28):46714678.

8. Stitzenberg KB, Wong YN, Nielsen ME, Egleston BL, Uzzo RG. Trends in radical prostatectomy: centralization, robotics, and access to urologic cancer care. Cancer. 2012;118(1):54-62.

9. Birkmeyer JD, Stukel TA, Siewers AE, Goodney PP, Wennberg DE, Lucas FL. Surgeon volume and operative mortality in the United States. N Engl J Med. 2003;349(22):2117-2127.

10. Birkmeyer JD, Dimick JB, Staiger DO. Operative mortality and procedure volume as predictors of subsequent hospital performance. Ann Surg. 2006;243(3):411-417.

11. Christians KK, Evans DB. Additional support for neoadjuvant therapy in the management of pancreatic cancer. Ann Surg Oncol. 2015;22(6):1755-1758.

12. Evans DB, George B, Tsai S. Non-metastatic Pancreatic Cancer: Resectable, Borderline Resectable, and Locally AdvancedDefinitions of Increasing Importance for the Optimal Delivery of Multimodality Therapy. Ann Surg Oncol. 2015;22(11):34093413.

13. Lassen K, Coolsen MM, Slim K, et al. Guidelines for perioperative care for pancreaticoduodenectomy: Enhanced Recovery After Surgery (ERAS(R)) Society recommendations. World J Surg. 2013;37(2):240-258.

14. Kehlet H. Multimodal approach to control postoperative pathophysiology and rehabilitation. $\mathrm{Br} \mathrm{J}$ Anaesth. 1997;78(5):606-617.

15. Ji HB, Zhu WT, Wei Q, Wang XX, Wang HB, Chen QP. Impact of enhanced recovery after surgery programs on pancreatic surgery: A meta-analysis. World J Gastroenterol. 2018;24(15):1666-1678.

16. Daniel SK, Thornblade LW, Mann GN, Park JO, Pillarisetty VG. Standardization of perioperative care facilitates safe discharge by postoperative day five after pancreaticoduodenectomy. PLoS One. 2018;13(12):e0209608.

17. Braga M, Pecorelli N, Ariotti R, et al. Enhanced recovery after surgery pathway in patients undergoing pancreaticoduodenectomy. World J Surg. 2014;38(11):29602966.

18. Coolsen MM, Bakens M, van Dam RM, Olde Damink SW, Dejong $\mathrm{CH}$. Implementing an enhanced recovery program after pancreaticoduodenectomy in elderly patients: is it 
feasible? World J Surg. 2015;39(1):251-258.

19. Lassen $\mathrm{K}$, Ljungqvist $\mathrm{O}$, Dejong $\mathrm{CH}$, et al. Pancreaticoduodenectomy: ERAS recommendations. Clin Nutr. 2013;32(5):870-871.

20. Kagedan DJ, Ahmed M, Devitt KS, Wei AC. Enhanced recovery after pancreatic surgery: a systematic review of the evidence. HPB (Oxford). 2015;17(1):11-16.

21. Christians KK, Tsai S, Tolat PP, Evans DB. Critical steps for pancreaticoduodenectomy in the setting of pancreatic adenocarcinoma. J Surg Oncol. 2013;107(1):33-38.

22. Bassi C, Marchegiani G, Dervenis C, et al. The 2016 update of the International Study Group (ISGPS) definition and grading of postoperative pancreatic fistula: 11 Years After. Surgery. 2017;161(3):584-591.

23. Wente MN, Veit JA, Bassi C, et al. Postpancreatectomy hemorrhage (PPH): an International Study Group of Pancreatic Surgery (ISGPS) definition. Surgery. 2007;142(1):20-25.

24. Malleo G, Crippa S, Butturini G, et al. Delayed gastric emptying after pylorus-preserving pancreaticoduodenectomy: validation of International Study Group of Pancreatic Surgery classification and analysis of risk factors. HPB (Oxford). 2010;12(9):610-618.

25. Callery MP, Pratt WB, Kent TS, Chaikof EL, Vollmer CM, Jr. A prospectively validated clinical risk score accurately predicts pancreatic fistula after pancreatoduodenectomy. J Am Coll Surg. 2013;216(1):1-14.

26. Zouros E, Liakakos T, Machairas A, Patapis P, Agalianos C, Dervenis C. Improvement of gastric emptying by enhanced recovery after pancreaticoduodenectomy. Hepatobiliary Pancreat Dis Int. 2016;15(2):198-208.
27. Dai J, Jiang Y, Fu D. Reducing postoperative complications and improving clinical outcome: Enhanced recovery after surgery in pancreaticoduodenectomy - A retrospective cohort study. Int J Surg. 2017;39:176-181.

28. Zhang L, Liao Q, Zhang T, Dai M, Zhao Y. Blood Transfusion is an Independent Risk Factor for Postoperative Serious Infectious Complications After Pancreaticoduodenectomy. World J Surg. 2016;40(10):2507-2512.

29. Brooks AD, Marcus SG, Gradek C, et al. Decreasing length of stay after pancreatoduodenectomy. Arch Surg. 2000;135(7):823-830.

30. Kunstman JW, Fonseca AL, Ciarleglio MM, Cong X, Hochberg A, Salem RR. Comprehensive analysis of variables affecting delayed gastric emptying following pancreaticoduodenectomy. J Gastrointest Surg. 2012;16(7):1354-1361.

31. Gaignard E, Bergeat D, Courtin-Tanguy L, et al. Is systematic nasogastric decompression after pancreaticoduodenectomy really necessary? Langenbecks Arch Surg. 2018;403(5):573580 .

32. Kunstman JW, Klemen ND, Fonseca AL, Araya DL, Salem RR. Nasogastric drainage may be unnecessary after pancreaticoduodenectomy: a comparison of routine vs selective decompression. J Am Coll Surg. 2013;217(3):481488.

33. Elliott IA, Chan C, Russell TA, et al. Distinction of Risk Factors for Superficial vs Organ-Space Surgical Site Infections After Pancreatic Surgery. JAMA Surg. 2017;152(11):1023-1029.

34. Bozzetti F, Mariani L. Perioperative nutritional support of patients undergoing pancreatic surgery in the age of ERAS. Nutrition. 2014;30(11-12):1267-1271. 